\title{
The Development of Decision Support System for Production of Layer
}

\author{
Jianhua Xiao, Hongbin Wang, Luyi Shi, Mingzhe Lv, and Haikun Ma \\ College of Veterinary Medicine, Northeast Agricultural University, \\ Harbin 150030, China
}

\begin{abstract}
The Decision Support System for Production of Commercial Layer was developed based on the demand analysis. VB .NET 2005 and SQL SERVER 2000 was used as main methods for system development. With this system, the data of alternation, growth, breeding, environment, immune for layer production can be recorded, edited, analyzed and be given by a report forms. With this system, the production efficiency can be raised.
\end{abstract}

Keywords: layer, DSS.

\section{Introduction}

For a large-scale layer enterprise, there must be many batches of chickens kept at the same time. And there must be many differences for observed indicators among those batches. Therefore, it was very difficult to ensure that each chicken goes according to plan production. This problem can be solved by developing one decision support system for production of commercial layer. In terms of computer software for layer production, one computer software for breeding was developed by Cai Juandeng, breeding data can be treated and various kinds of genetic parameters can be obtained by this soft [1]. One management system for chicken breeding data was developed by Xiao Fan, which has the pedigree matching, hatching management, determination of egg, report query etc[2]. One system of data acquisition and input for egg production was developed in 1993 by Yang Ning using bar code technology and handheld computer technology [3]. One digital breeding platform for breeding pig was developed by Zhao ruixue [4]. One management information system for layer farm was developed by Wu Xiaohong [5]. In addition, the computer software is also used in retrospective [6], disease diagnosis [7] and so on. The Decision Support System for Production of Commercial Layer was developed in this study aimed at recording and analyzing the data of growth, feeding, egg production, environment, immunization, and disease, changes during brood period, Incubation period, and laying period and out. By management and statistical analysis of production data, the overall situation and problems can be controlled by managers with this system, and the objective, scientific decision can be made by managers. 


\section{Principle and Methods}

\subsection{The Production Flow-Sheet of Layer}

There are four stages during a production cycle of goods layers. They can be listed as follows: birth, incubation, egg, and out. The brood period is from its birth to six weeks. The incubation period is from the seventh to twentieth weeks. The egg period is from twenty to seventy-two weeks; then layer is out, different indicators need to be obtained in each period.

\subsection{The Development Methods and System Framework}

In general, one chicken farm is composed of several branches. moreover easy of use is required, and therefore the C/S framework was used in this system. One computer was set as server and the computer in every branch was installed client soft; to access the data in server by set the only IP address. The operating system for developed this soft was selected as windows XP, and Microsoft VB.NET 2005 was selected as development language[9], the excel component of Microsoft office was used to import and export data, the third party component Dotnet Charting was used to generate figure. Microsoft SQL Server 2000 was used as database.

\section{$3 \quad$ Result}

The system was divided into 6 modules based on the production processes of layer: the management of farm and house, data input, statistics and report forms, curve diagram, system management and help. The initial data of one batch of chickens was

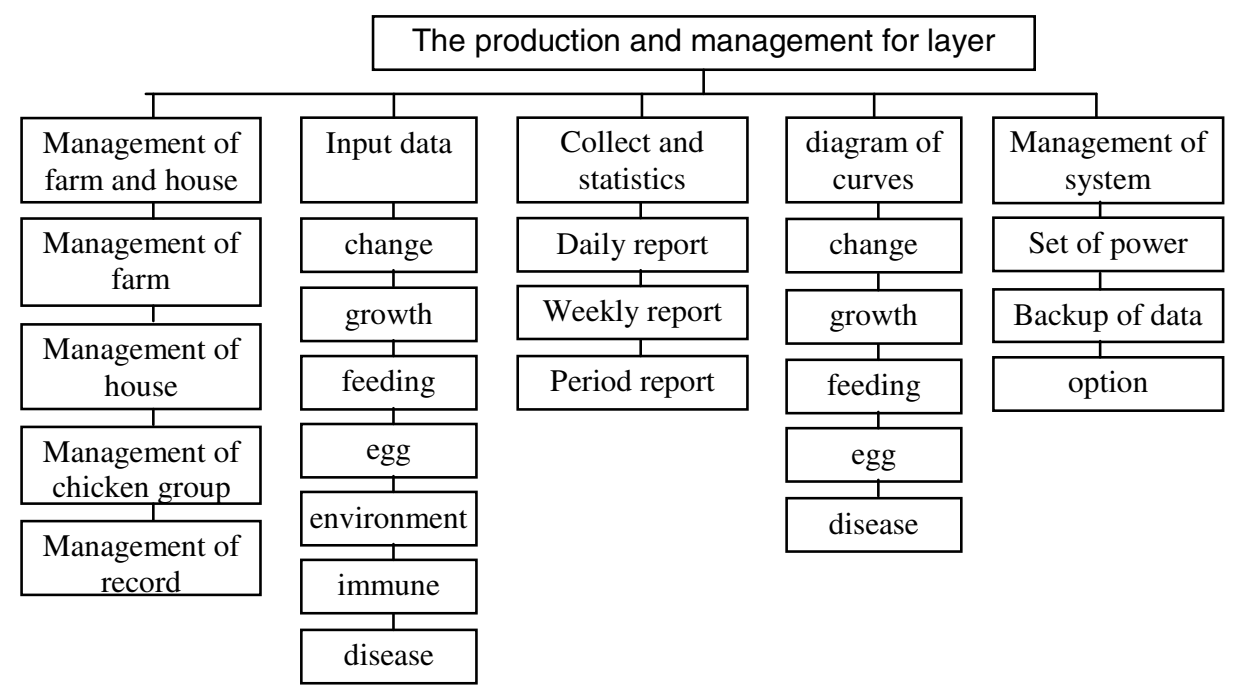

Fig. 1. The function and structure of production and management system for layer 
recorded after being introduced; the data generating in every production processes was record synchronously. All of data were done statistics in a statistics and report forms module, and also produced daily, weekly, periodic report. The function and structure of system can be found in figure 1 .

\subsection{The Introduction of One Batch of Chicken}

The basic data of one batch of chicken include information generated when they were introduced and information generated in every production stages. Those data mainly included serial number, species, source, approach date, born weight, quantity, stocking rate and survival rate after 24 weeks, date when increase light, egg date etc. because different species of chicken have different breeding standard, and those procedures such as increasing light and egg, mix chicken groups must be completed within the limited periods, therefore the standard of production was compiled in software, and the program will compute everyday according to this standard, the current status of chickens can be get, and the attention for change house, increase light, mix groups, etc. was gave to user automatically(fig. 2).

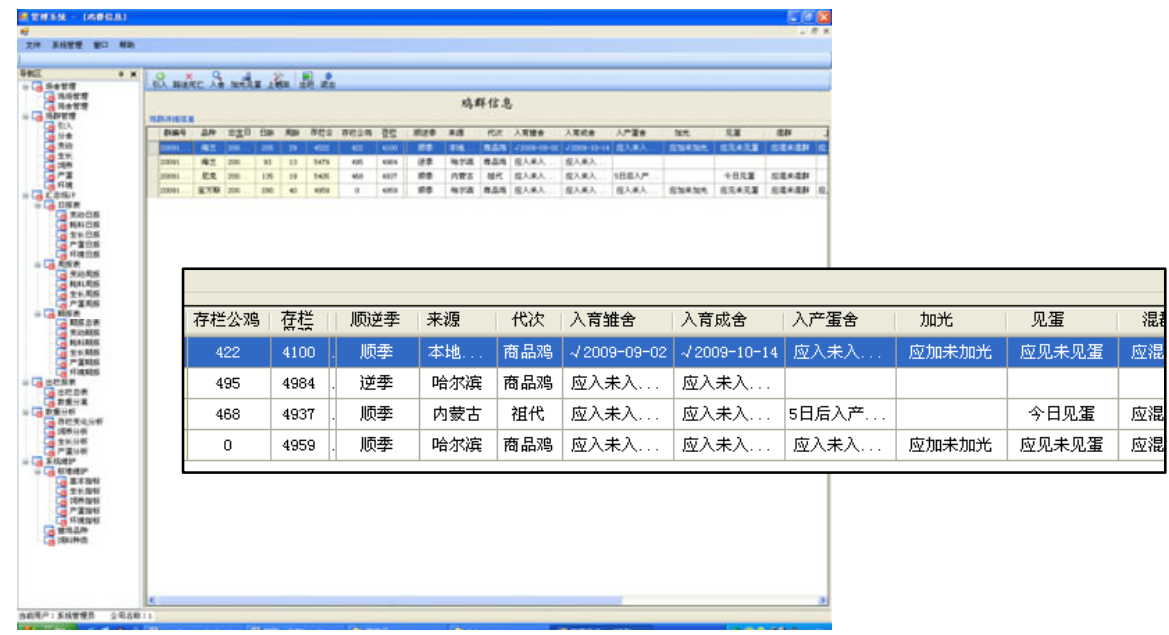

Fig. 2. The introduction of chickens and attentions in key production processes

The data can be modified at any time and export into excel table. The production data need to be backed up and separated when production cycle of one batch of chicken has closed, then the data of this batch of chicken can be deleted from database.

\subsection{The Management of Change Data for One Batch of Chicken}

Death, eliminate, marketing, output and input of chickens are main changes of one batch of chicken in production. These data and data of herbs must be recorded in to computer every day. In order to facilitate data recording, few data (such as death and eliminate etc.) need to be input and closing stock will be computed by numbers of yesterday automatically. By this ways, the efficiency would be improved 
significantly. The changes of data can be statistics, daily, weekly, and period report and figure of one phase can be generated in system. By these results, more scientific measure can be made by managers. By contrasted to standard, the conclusion of production can be got(fig. 3 ).

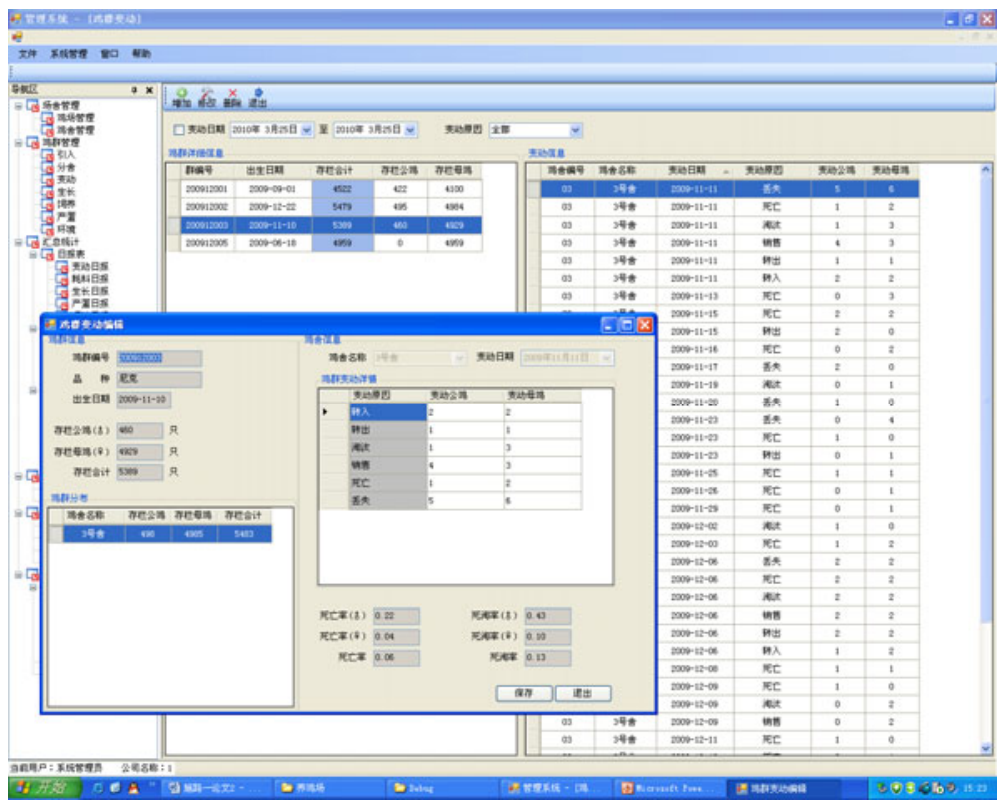

Fig. 3. The changes data of one batch of chicken

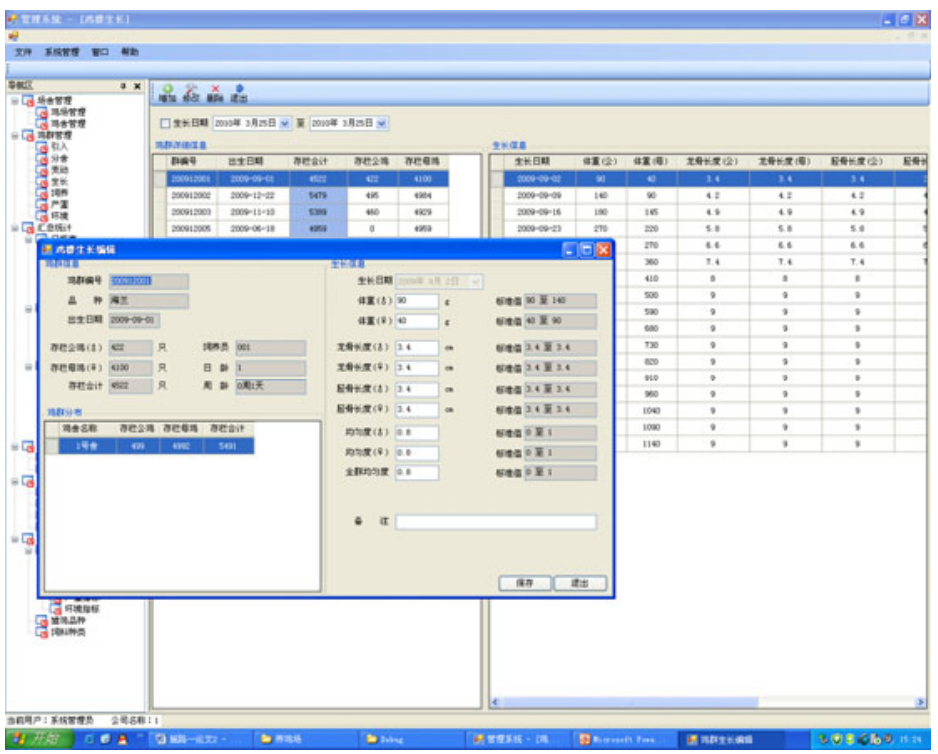

Fig. 4. The management of growth data for one batch of chicken 


\subsection{The Management of Growth Data for One Batch of Chicken}

The growth was the main observation for management during brood and incubation period. Sound skeletal structure is necessary for egg production. It is very important to monitor redords and analyzation of the chickens growth informotion. Only the weight of chicken at Pre-laying (from 21 to 28 week) need to be record weekly. Based on the general requirement of layer farm, body weight, uniformity, average uniformity, change of feather, length of keel, length of tibia need to be managed in this system. The growth data can be statistics, daily report, weekly report, report of one phase can be generated in system (fig.4).

\subsection{The Management of Feeding Data for One Batch of Chicken}

The feeding is associated with each period of production. The difference among those periods is the feed. Therefore feeding methods, the number of feed, feed consumption, water consumption, feeding time, difference between planning and the actual feed consumption, protein and metabolic energy intake and other related information need to be recorded in database. By comparing, the information whether the actual feed consumption has met the standards will be reminder to user. In addition, the feeding data can be statistics, daily report, weekly report, report of one phase and figure of changing can be generated in system. Thus managers can investigate the reason for insufficiency or excessive, and take effective measures timely (fig.5).

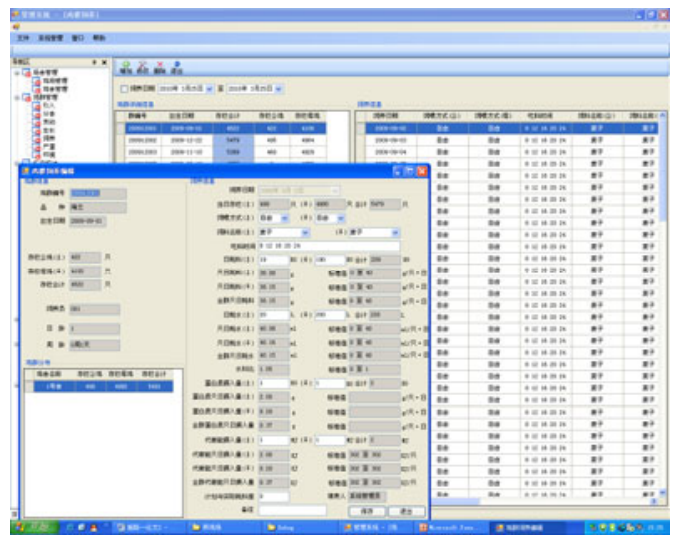

Fig. 5. The management of feeding data for one batch of chicken

\subsection{The Management of Production Data for One Batch of Chicken}

Egg number, number of unqualified eggs, fertilized eggs, ratio of feed to egg need to managed. Those data can be input from three routes: (1)input by manager directly, (2) generated from system by computed, (3)obtain from computer directly or generate from system daily. The number of hatching egg, number of goods egg, number of double-yolked egg, number of soft egg need to be recorded by manager daily. And 
laying rate, pass rate of hatching egg, ratio of commodity egg to total egg, double yellow egg ratio, ratio of soft eggs, broken egg rate, fertilization rate, hatching rate, etc generated from system by computed. Every data can be count(fig.6), and statistical graph can be generated(fig.7).

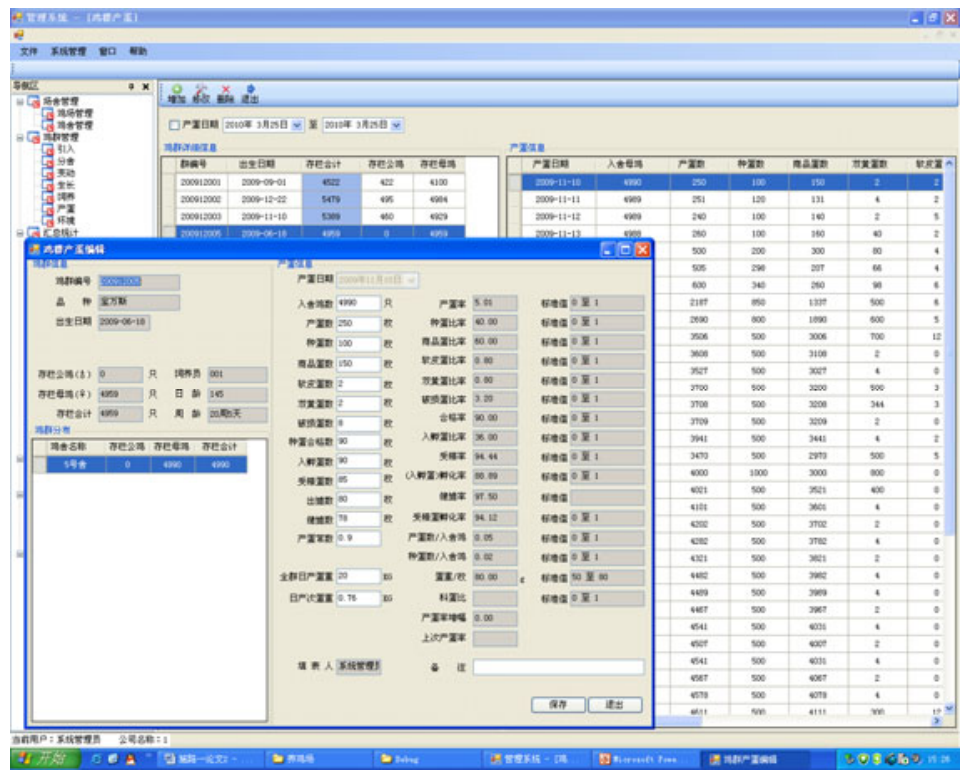

Fig. 6. The management of production data for one batch of chicken

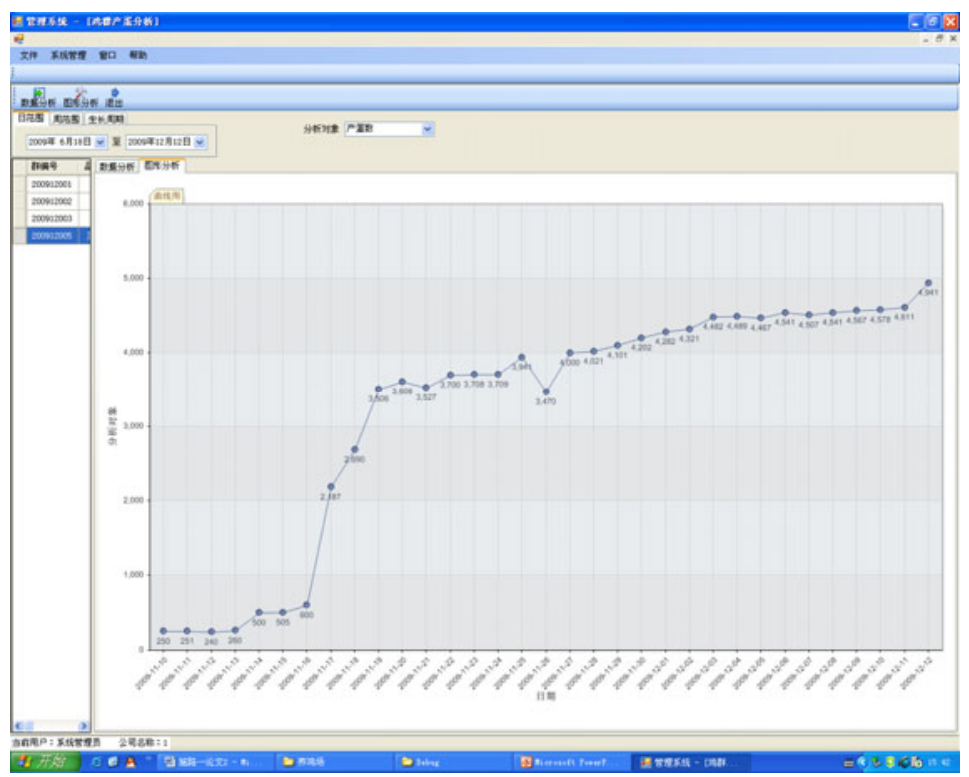

Fig. 7. The statistical graph of production data for one batch of chicken 


\section{Discussion}

\subsection{Data Inputting of Management System}

Too many items and large amounts of data are main factors that restrict the application of software in practices. Although some data can be recorded by auto technology, however, some data still be input manually. It is so important that reduce the input by hand and improve the efficiency. The reducing of input was considered in any parts of this system, besides the keying order of textbox was adjusted, many data can be computed by system automatically. Moreover, the association of items among different modules was enhanced in system, and user can find the data that he wanted in place where he wanted.

\subsection{The Intelligence of Management System}

Generally, many batches of chicken will be feed simultaneously in one large-scale farm, and different batches of chicken will be produced at different stages. Different items need to be managed for each batch of chicken. In order to improve the intelligence of system, the production information will be retrieved and computed by production standard every day, and the works need to be completed can be displayed in a form. The software can be used to look over new task.. By this way, the fault in will be reduced significantly.

\subsection{Decision Support for Production of Management System}

The main purpose that a manager using the software is to do statistics and analyzes production data, thereby finding out the problem during the production, so that making the work plans on the basis of current data. This quest was considered thoroughly when this system being designed and developed. The production data in every procedure can be statistics by day, week and period, and the statistical graph can be generated directly.

\section{References}

1. Cai, J., Tong, H.B.: The Application of Computer in Breed for Chicken. Shandong Poultry 5, 14-16 (1997)

2. Fan, X.: The Design and Application of Data Management System for High Quality Fowl Breeding. China Poultry 31, 4-7 (2009)

3. Yang, N., Shan, C., Li, J.: Research on Record System Without Paper for Fowl Egg Number. Journal of Chinese Animal Husbandry 29, 3-5 (1993)

4. Zhao, R., Zhao, P., Gong, C.: Research on Digital Cultivation Technique Platform for Goods Layer. Heilongjiang Animal Husbandry and Veterinary 12, 47-48 (2006) 
5. Wu, X., Bin, S.: The Computer Network Management for Modern Fowl Fram. Journal of Zhongkai Agrotechnical College 6, 53-59 (1993)

6. Zhao, J.: The Analysis for Application Current Situation of Trace System for Chicken Quality in China. Chinese Journal of Animal Science 47, 45-48 (2011)

7. $\mathrm{Xu}$, J.: The Diagnosis Expert System for Common Disease of Fowl. Chinese Journal of Veterinary Medicine 18, 40-41 (1992)

8. Yin, H.: Useful Course of Studies for Visual Basic.NET. China Railway Publishing House, Beijing (2003) 